\title{
Role of Stem Cells in the Anti-tumor Effects of the Human Amniotic Membrane
}

\author{
Mahsa Khayat-Khoei* \\ Department of Cardiology, Baylor CHI St. Luke's Health, Houston, Texas, USA \\ *Corresponding author: Mahsa Khayat-Khoei, Department of Cardiology, Baylor CHI St. Luke's Health, Houston, Texas, USA, Tel: 713 909-0996; E-mail: \\ mahsa.khoei@dr.com
}

Received date: Sep 28, 2015, Accepted date: Sep 30, 2015; Published date: Oct 02, 2015

Copyright: ( 2015 Khayat-Khoei M. This is an open-access article distributed under the terms of the Creative Commons Attribution License, which permits unrestricted use, distribution, and reproduction in any medium, provided the original author and source are credited.

\section{Editorial}

Many novel ideas have emerged in cancer management, among which using stem cells has been one of the highlights. Stem cells have been used to control and treat the blood and immune system damages due to cancer cells invasion or side effects of chemotherapy and radiation therapy [1]. Several studies have focused on the role of cancer stem cells $[1,2]$, as well as cancer gene therapy mainly in neural stem cells [3] and stem cell transplantation [4].

One of the exciting new ideas that have been recently proposed in the field of cancer therapy using stem cells is the possible application of Amniotic Membrane (AM) in controlling cancer [5]. Even though there are published data on anti-cancer properties of the AM, the exact molecular mechanism of these effects are not yet clear and there are ongoing researches in the field that we aim to summarize and review here.

Amniotic Membrane, the innermost layer of fetal membrane, is a special structure that functions as a barrier that separates the fetus from the maternal tissue and provides an anatomically, physiologically and immunologically privileged space for the future baby to thrive. This membrane is proven to have special characteristics such as antiangiogenic, immunoregulatory and pro-apoptotic $[5,6]$ as well as antimicrobial, anti-fibrosis, and low immunogenicity properties [7]. The $\mathrm{AM}$ owes these properties to its unique histologic features of having two different types of stem cells: A single layer of Amniotic Epithelial Stem Cells (AESCs) on a thick basement membrane, and Amniotic Mesenchymal Stem Cells (AMSCs) in an avascular stroma. Seo and colleagues were the first scientists who hypothesised the possible anticancer properties of the AM in 2008 [8]. They suggested pro-apoptotic activities as well as anti-angiogenic and immunoregulatory capacities for the AM and argued that these properties can be applicable in cancer therapy. The hypothesised anti-cancer effects of the AM was further discussed and evaluated by Niknejad et al. [5,9], Jiao et al. [10] and Magatti et al. [11].

Providing a proper blood supply for tumor cells is a key element in tumor growth and metastasis [5] and the AM is believed to possess anti-angiogenesis properties $[5,6,8,9,12]$. There are reports of antiangiogenic factors such as collagen XVIII, thrombospondin-1, interleukin (IL)-1 receptor antagonist, IL-10 and tissue inhibitor of metalloprotease (TIMP-1, TIMP-2, TIMP-3, and TIMP-4) being mainly produced by the AM-derived Epithelial Stem Cells [12]. On the other hand, the Amniotic Mesenchymal Stem Cells secrete factors with angiogenesis promoting activity such as intravascular adhesion molecule, growth-related oncogene, IL-6, IL-8, migration inhibitory factor and monocyte chemoattractant protein-1 [13]. Niknejad et al. evaluated the role of AM in angiogenesis more thoroughly using an aorta ring assay and demonstrated different angiogenesis properties for $\mathrm{AM}$ based on the attached stem cells on the membrane. In the presence of AESCs, no capillary formation was reported even though AMSCs were present; whereas the AM could induce angiogenesis when the AESCs were scraped and only the AMSCs were remaining $[5,6]$. This suggests that the AESCs are the main cells responsible for the antiangiogenesis properties of the AM.

The anticancer effects of the Amniotic Membrane are also due to its anti-proliferative properties. Magatti and colleagues recently reported that the AM can induce cell cycle arrest in G0/G1 phase by unknown soluble factors [11]. These factors, produced by the AM stem cells, can down-regulate expression of cancer cells genes associated with cell cycle progression such as cyclin-dependent kinases (CDK 2, 4 and 6) and cyclins (Cyclin D2, E1 and H) [11]. Another possible factor that might contribute to the antiproliferative effects of the AM is inhibition of heat shock protein 90 (HSP90) by amniotic derived substances [14]. HSP90 is an adenosine triphosphate (ATP)-dependent molecular chaperone that acts as a reversal to cancer cell destruction by having opposite regulatory effects on most or all phases of cell cycle. Hsp90 affects the function of major regulators of cell cycle, by directly or indirectly up-regulating of cyclins $\mathrm{B}, \mathrm{D}$ and $\mathrm{E}$ as well as cyclin dependent kinases including CDKs $1,2,4$ and 6 . It can enhance angiogenesis in new cancer cell formation and protect cancer cells by blocking apoptosis and cell cycle arrest mechanisms. Therefore inhibition of HSP90 is favorable in cancer therapy as it can lead to inhibition of CDK 2 and cyclin $\mathrm{E}$ and enhance cell cycle arrest in G1/S boundary [15]

The final mechanism that is proposed in favor of the anticancer characteristics of the AM is induction of apoptosis by this membrane. Jiao et al. reported decreased expression of $\mathrm{Bcl}-2$ and high levels of apoptoticmarkers Bax, caspase- 3 and caspase- 8 in glioma cells after treatment with the Amniotic Stem Cells [10]. The same significant increase in caspase- 3 and caspase- 8 was reported in another study on HeLa and MDA-MB-231 cancer cell lines treated with Amniotic Epithelial Stem Cells [5]. The results were confirmed by publishing series of multi-media data showing cancer cell degradation and reduction in cancer cell motility after treatment with supernatant derived from the AESCs [5].

In conclusion, the Amniotic Membrane can have possible applications in cancer therapy through its anti-proliferative and pro apoptotic properties. Considering the fact that anti-angiogenesis property of the AM was mainly demonstrated in the presence of Amniotic Epithelial Stem Cells, these cells rather than the whole AM, can be candidates for new therapies to control cancer cell growth and metastasis. Yet more research is necessary to determine the exact mechanism and substance responsible for these anticancer effects. 
Citation: Khayat-Khoei M (2015) Role of Stem Cells in the Anti-tumor Effects of the Human Amniotic Membrane. J Cytol Histol 6: e117. doi:

Page 2 of 2

\section{References}

1. Sagar J, Chaib B, Sales K, Winslet M, Seifalian A (2007) Role of stem cells in cancer therapy and cancer stem cells: a review. Cancer Cell Int 7: 9 .

2. Koch U, Krause M, Baumann M (2010) Cancer stem cells at the crossroads of current cancer therapy failures--radiation oncology perspective. Semin Cancer Biol 20: 116-124.

3. Ahmed AU, Alexiades NG, Lesniak MS (2010) The use of neural stem cells in cancer gene therapy: predicting the path to the clinic. Curr Opin Mol Ther 12: 546-552.

4. Moreau P, Avet-Loiseau H, Harousseau JL, Attal M (2011) Current trends in autologous stem-cell transplantation for myeloma in the era of novel therapies. J Clin Oncol 29: 1898-1906.

5. Niknejad H, Khayat-Khoei M, Peirovi H, Abolghasemi, H (2014) Human amniotic epithelial cells induce apoptosis of cancer cells: a new antitumor therapeutic strategy. Cytotherapy 16: 33-40.

6. Niknejad H, Paeini-Vayghan G, Tehrani FA, Khayat-Khoei M, Peirovi H (2013) Side dependent effects of the human amnion on angiogenesis. Placenta 34: 340-345.

7. Niknejad H, Peirovi H, Jorjani M, Ahmadiani A, Ghanavi J, et al. (2008) Properties of the amniotic membrane for potential use in tissue engineering. Eur Cell Mater 15: 88-99.
8. Seo JH, Kim YH, Kim JS (2008) Properties of the amniotic membrane may be applicable in cancer therapy. Med Hypotheses 70: 812-814.

9. Niknejad H, Khayat-Khoei M, Peirovi H (2012) Inhibition of MMPs might increase anticancer properties of amniotic epithelial cells. Med Hypotheses 78: 690-691.

10. Jiao H, Guan F, Yang B, Li J, Song L, et al. (2012) Human amniotic membrane derived-mesenchymal stem cells induce C6 glioma apoptosis in vivo through the Bcl-2/caspase pathways. Mol Biol Rep 39: 467-473.

11. Magatti M, De Munari S, Vertua E, Parolini O (2012) Amniotic membranederived cells inhibit proliferation of cancer cell lines by inducing cell cycle arrest. J Cell Mol Med 16: 2208-2218.

12. Hao Y, Ma DH, Hwang DG, Kim WS, Zhang F (2000) Identification of antiangiogenic and antiinflammatory proteins in human amniotic membrane. Cornea 19: 348-352.

13. Hwang JH, Shim SS, Seok OS, Lee HY, Woo SK, et al. (2009) Comparison of cytokine expression in mesenchymal stem cells from human placenta, cord blood, and bone marrow. J Korean Med Sci 24: 547-554.

14. Trepel J, Mollapour M, Giaccone G, Neckers L (2010) Targeting the dynamic HSP90 complex in cancer. Nat Rev Cancer 10: 537-549.

15. Burrows F, Zhang H, Kamal A (2004) Hsp90 activation and cell cycle regulation. Cell Cycle 3: 1530-1536. 